\title{
MODELAGEM DO CRESCIMENTO DE BACTÉRIAS ÁCIDO LÁCTICAS DURANTE A VIDA DE PRATELEIRA DE PRESUNTO COZIDO FATIADO EMBALADO A VÁCUO TRATADO COM EXTRATO SUPERCRÍTICO DE Rosmarinus officinalis.
}

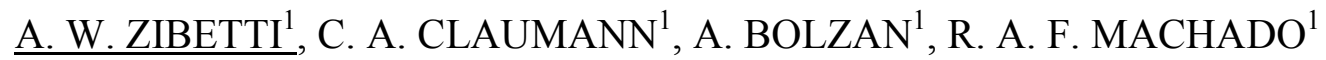 \\ ${ }^{1}$ Universidade Federal de Santa Catarina, Departamento de Engenharia Química e Engenharia de \\ Alimentos \\ E-mail para contato: azibetti@gmail.com
}

\begin{abstract}
RESUMO - A aplicação de extratos naturais em produtos à base de carne, para prolongar sua vida de prateleira, tem sido investigada. Presunto cozido fatiado embalado a vácuo (PCF) pode apresentar alta população de bactérias ácido lácticas (BAC) durante o armazenamento refrigerado, o que pode limitar a sua vida de prateleira. Foi avaliada a vida de prateleira do presunto de cozido embalado a vácuo com adição de extratos naturais. Foram determinados os efeitos do extrato supercrítico e óleo essencial hidrodestilado de Rosmarinus off. em comparação com o produto controle. O crescimento de BAC foi monitorado durante 40 dias no PCF armazenado a $7{ }^{\circ} \mathrm{C}$. Foi definido como o critério limitador da vida de prateleira $10^{7} \mathrm{UFC} / \mathrm{g}$ de produto. Observou-se o aumento da vida de prateleira das amostras tratadas, em média de 8 dias. Foram ajustados modelos matemáticos microbiológicos preditivos aos dados experimentais (Gompertz modificado GMZ, logístico modificado LMZ e LMCP).
\end{abstract}

\section{INTRODUÇÃO}

Os vegetais são de longe as fontes naturais mais bem estudadas, pois contém uma variedade enorme de compostos ativos que podem ser utilizados na indústria de alimentos como compostos funcionais ou nutracêuticos. A crescente demanda por produtos cárneos seguros tem impulsionado a indústria e a academia em pesquisas para aprimorar a preservação desses alimentos, sem o uso de conservantes artificiais. Carnes e derivados são altamente propensos a contaminação microbiana, uma vez que são ricos em nutrientes essenciais e perecíveis na natureza (JAYASENA; JO, 2013).

Os antioxidantes e antimicrobianos presentes em algumas espécies vegetais são compostos que, quando presentes em alimentos, em baixa concentração, comparado a um substrato, claramente atrasa ou previne a sua oxidação e o crescimento de deteriorantes ou contaminantes. Os extratos de alecrim (Rosmarinus officinalis) possuem comprovada atividade antioxidante e antimicrobiana (GENENA et al., 2008; ZIBETTI; BOLZAN; MARANGONI, 2009). A microbiologia preditiva tem sido extensivamente utilizada para modelar o crescimento de bactérias, como uma função de fatores intrínsecos e extrínsecos com intuito de prever a vida de prateleira de produtos alimentícios. Para 
tanto, diferentes modelos foram propostos levando em consideração diferentes fatores. As equações sigmoidais, como logística e Gompertz modificados, têm sido amplamente utilizadas para descrever o crescimento de bactérias, dentre elas as bactérias láticas (LONGHI et al., 2013; SLONGO et al., 2009).

As bactérias ácido láticas (BAC) são encontradas naturalmente em muitos produtos cárneos embalados a vácuo e armazenados sobre refrigeração. Essas bactérias encontradas no presunto cozido fatiado embalado a vácuo são responsáveis pela sua deterioração e consequente redução da sua vida de prateleira (VERCAMMEN et al., 2011). O uso de modelos matemáticos capazes de predizer o crescimento microbiano ao longo do armazenamento do presunto cozido fatiado embalado à vácuo é uma ferramenta importante para avaliar a influência da adição do extrato natural obtido do alecrim no aprimoramento da vida de prateleira desse produto. Um número reduzido de trabalhos tem avaliado a influência da adição de extratos naturais na vida de prateleira de um produto cárneo comercial. Desta forma o objetivo principal deste trabalho foi o de investigar a influência do extrato supercrítico obtido do alecrim (Rosmarinus officinalis) na vida de prateleira do presunto cozido fatiado embalado à vácuo armazenado a $7{ }^{\circ} \mathrm{C}$ com tratamento (adição do extrato natural SC e HD) e comparado ao controle (sem tratamento). Os modelos preditivos primários foram ajustados aos dados experimentais a fim de verificarem sua aplicabilidade na previsão da vida de prateleira (tempo para atingir o limite de $\log 10^{7}$ $\mathrm{UFC} / \mathrm{g}$ de produto) e verificar a influência dos tratamentos nas respostas preditivas. Uma análise criteriosa dos parâmetros foi aplicada para identificar as possíveis alterações no crescimento de BAC no PCF.

\section{MATERIAIS E MÉTODOS}

\subsection{Presunto cozido fatiado embalado a vácuo}

O presunto cozido, composto basicamente de carne suína (pernil suíno), proteína de soja, água, sal e açúcar foi fabricado e embalado a vácuo de acordo com o memorial descritivo da empresa comercial. As amostras foram tratadas com extrato supercrítico (SC) e oléo essencial hidrodestilado (HD) de alecrim. As amostras foram retiradas de sua embalagem original em uma cabine de fluxo laminar onde foram aplicados os tratamentos na superfície do mesmo, sendo: SC - adição de 0,004 g extrato supercrítico/g produto; HD adição de 0,004 g óleo essencial hidrodestilado/g produto; CONTROLE - sem adição. Após a aplicação dos tratamentos as amostras foram re-embaladas, submetidas a vácuo e armazenadas na temperatura de $7^{\circ} \mathrm{C}$ (considerado uma temperatura de "abuso" no armazenamento desses produtos). As análises microbiológicas foram realizadas ao longo da vida de prateleira do produto, nos tempos 0, 10, 20, 30 e 40 dias, em triplicatas (SC, HD e CONTROLE), totalizando 45 amostras. $\mathrm{O}$ crescimento de BAC foi monitorado ao longo do tempo. Todas as análises foram realizadas em laboratório (LABCAL - UFSC) credenciado junto ao MAPA e habilitado pela ANVISA, e a metodologia utilizada segundo foi segundo a American Public Health Association (DOWNES; ITO; ASSOCIATION, 2001).

\subsection{Procedimentos de extração}

Foram realizados dois procedimentos de extração para obtenção dos extratos naturais de 
alecrim: extração supercrítica (SC) e hidrodestilação (HD). A caracterização do extrato supercrítico (SC) e do óleo essencial (hidrodestilado, HD) foi realizada via GC-MS para identificação dos compostos e posteriormente via GC-FID para qualificação dos extratos em termos da área relativa do pico. A comparação foi realizada somente comparando os compostos com mesmo tempo de retenção.

Extração supercrítica: Para obtenção do extrato supercrítico de alecrim foi utilizada uma unidade piloto, denominada SFE-I, equipamento e o procedimento de extração já foram descritos em (WÜST ZIBETTI et al., 2013).

Hidrodestilação: $O$ processo de hidrodestilação foi realizado utilizando um aparelho tipo Clevenger, com um balão de 5 Litros e manta de aquecimento. Aproximadamente $300 \mathrm{~g}$ de alecrim seco e moído foram utilizados em cada batelada, com duração de $180 \mathrm{~min}$, ou até que o volume de óleo essencial coletado se mantivesse constante.

\subsection{Modelagem}

Para o presente estudo foram considerados os modelos primários, logístico modificado por Zwietering (LMZ), Gompertz modificado por Zwietering (GMZ) e logístico modificado por Corradini e Peleg (LMCP). Zwietering et al. (1990) re-parametrizaram a equação original de Gompertz (Equação 1) e a equação Logística (Equação 2) para descrever o crescimento microbiano isotermicamente, considerando a taxa específica de crescimento máximo $\left(\mu_{\text {máx }}\right)$, o tempo da fase lag $(\lambda)$ e a contagem máxima de células alcançada $(A)$ como parâmetros cinéticos. A resposta é normalizada em relação ao logaritmo natural da contagem inicial de bactérias $\left(N_{0}\right)$, sendo $N(t)$ a contagem de bactérias no tempo $(t)$ (CORRADINI; PELEG, 2005; ZWIETERING et al., 1990):

$$
\begin{gathered}
\log \left(\frac{N(t)}{N_{0}}\right)=A \exp \left\{-\exp \left(\frac{\exp (1) \mu_{\max }}{A}(\lambda-t)+1\right)\right\} \\
\log \left(\frac{N(t)}{N_{0}}\right)=\frac{A}{1+\exp \left[\left(\frac{4 \mu_{\max }}{A}\right)(\lambda-t)+2\right]}
\end{gathered}
$$

Corradini e Peleg (2005) modificaram o modelo Logístico (Equação 3) para representar os dados de crescimento microbiano. Onde $A, k$ e $t_{c}$ são os parâmetros empíricos do modelo, $t_{c}$ é termo que regula o deslocamento do ponto de inflexão da curva, $k$ representa a fase de crescimento constante dos valores na curva (taxa de crescimento) e $A$ especifica a amplitude da curva sigmoide.

$$
\log \left(\frac{N(t)}{N_{0}}\right)=\frac{A}{1+\exp \left[k\left(t_{c}-t\right)\right]}-\frac{A}{1+\exp \left(k t_{c}\right)}
$$

As estimativas dos parâmetros foram feita por regressão não linear (otimização não linear com restrição nos parâmetros, software Matlab $\left.{ }^{\circledR}\right)$. Foram estabelecidas as mesmas restrições para cada um dos parâmetros de cada modelo avaliado. Para os parâmetros $A\left(\ln \left(N / N_{0}\right)\{0 \leq A \leq 25\}\right.$; $\mu_{\max }$ e $k\left(h^{-1}\right)\left\{0 \leq \mu_{\max }\right.$ ou $\left.k \leq 1\right\}$ e $\lambda$ e $t_{c}(h)\left\{0 \leq \lambda\right.$ ou $\left.t_{c} \leq 500\right\}$. As restrições foram 
escolhidas após avaliações exploratórias e via gráfica.

\subsection{Análise estatística}

A comparação entre os modelos e os seus respectivos parâmetros foram avaliados pelos os índices estatísticos: raiz do erro médio quadrático (RMSE) e o coeficiente de determinação $\left(\mathrm{R}^{2}\right)$. Para quantificar o erro cometido ao estimar os parâmetros dos modelos preditivos de crescimento microbiológico e estimar a sua distribuição para determinação do intervalo de confiança (95\%) foi utilizada a metodologia de boostraping. Enquanto os métodos assintóticos dependem do pressuposto ou conhecimento do comportamento da distribuição, que nem sempre é alcançada, essa metodologia é uma forma eficiente para obtenção dos intervalos de confiança sem a necessidade dos pressupostos sobre a distribuição do estimador (THAI et al., 2013).

\section{Método de Bootstrap}

O princípio do método de bootstrap é gerar repetidas pseudo-amostras distribuídas de acordo com a mesma distribuição da amostra original. A distribuição original pode ser substituída pela distribuição empírica da amostra, que é conhecida como bootstrap não paramétrica. $\mathrm{O}$ intervalo de confiança bootstrap (IC 95 \%) foi construído pelos percentis da distribuição amostral dos parâmetros $(2,5 \%$ até $97,5 \%)$. O algoritmo detalhado para a geração das pseudo-amostras bootstrap não paramétrica está descrito em (THAI et al., 2013). Foram utilizadas boostrap com $B=3000$ reamostragens para cada modelo testado, totalizando 9000 simulações.

\section{RESULTADOS}

Observa-se que o óleo essencial (HD) apresentou uma maior quantidade de $\alpha$-pineno (12\%) quando comparado aos extratos supercríticos $(3 \%)$. Pode-se observar também que os extratos de Rosmarinus officinalis, testados nesse trabalho, apresentam uma grande quantidade de canfora, superior a $40 \%$. Os principais constituintes encontrados nas amostras, foram, respectivamente para $\mathrm{SC}$ e HD, o $\alpha$-pineno (3 e $12 \%$ ), $\beta$-myrceno (5 e $2 \%$ ), 1,8-cineol (13 e $7 \%$ ), linanol (2 e $1 \%)$, canfora (41 e $44 \%$ ), borneol (2 e 3\%), $\alpha$-terpineol (3 e $5 \%$ ), verbenona (15 e $8 \%$ ) e o $\beta$-cariofileno (8 e $7 \%$ ). A porcentagem aqui demonstrada se refere à área do composto normalizada com à área total dos compostos identificados no cromatograma, e foi utilizado o mesmo número para as análises em comparação. Observou-se que o extrato supercrítico apresentou uma maior quantidade de sesquiterpenos quando comparados ao óleo essencial obtido por hidrodestilação, isso se deve ao método de extração utilizado.

O ajuste dos três modelos testados aos dados experimentais obtiveram coeficientes de determinação $\left(R^{2}\right)$ superiores a 0,98 e $R M S E$ iguais ou abaixo 0,72 . Observa-se na Figura 1 os modelos ajustados aos dados experimentais de crescimento de bactérias acido láticas (BAC) das amostras controle, tratada com extrato hidrodestilado (HD) e com extrato supercrítico (SC), armazenadas a $7{ }^{\circ} \mathrm{C}$. Na Tabela 1 são apresentados os valores obtidos para os parâmetros biológicos dos modelos de Gompertz modificado (GMZ) e Logístico modificado (LMZ) sendo eles A, $\mu_{\text {máx }}, \lambda$, e os valores dos parâmetros empíricos do modelo Logístico modificado por Corradini e Peleg (LMCP), 
$\mathrm{A}, \mathrm{k}, \mathrm{t}_{\mathrm{c}}$. Os valores dos parâmetros bootstrap $\left(\hat{\theta}_{\mathrm{b}}^{*}\right)$ foram obtidos a partir das pseudo-amostras geradas pelo método de bootstrap $(B=3000)$, expressos como média \pm desvio padrão $\left(\hat{\theta}_{B} \pm D_{B}\right)$ e seus respectivos intervalos de confiança (IC 95 \%). O parâmetro de maior interesse para avaliação da vida de prateleira do produto analisado foi a taxa de crescimento da bactéria, explicitada pelos modelos como $\mu_{\text {máx }}$ e $\mathrm{k}$.

Para as triplicatas puras, verificou-se pela análise de variância $(\alpha=0,05)$ e testes multicomparação (Tukey), uma diferença significativa entre as taxas de crescimento das amostras tratadas com os extratos (HD e SC) e o controle, sendo as maiores taxas encontradas na amostra controle $(0,079 \pm 0,037-\mathrm{GMZ} ; 0,065 \pm 0,027 \mathrm{LMZ} ; 0,019 \pm 0,011 \mathrm{LMCP})$ quando comparadas as tratadas com extrato supercrítico SC $(0,021 \pm 0,001-\mathrm{GMZ} ; 0,023 \pm 0,001 \mathrm{LMZ} ; 0,005 \pm 0,001 \mathrm{LMCP})$ e óleo essencial HD $(0,040 \pm 0,004-\mathrm{GMZ} ; 0,04 \pm 0,003 \mathrm{LMZ} ; 0,012 \pm 0,001 \mathrm{LMCP})$. Para a taxa específica de crescimento máximo $\left(\mu_{\text {máx }}\right)$ a maior sensibilidade do parâmetro foi encontrada no modelo de Gompertz com coeficiente de variação de $47 \%$. Os valores obtidos para a $\mu_{\text {máx }}$ nas amostras controle foram $15 \%$ menor para o modelo LMZ e $75 \%$ menor para LMCP quando comparados ao GMZ. Pela análise da distribuição amostral do parâmetro $\mu_{\text {máx }}$ obtida pela metodologia bootstrap pode-se verificar que para o modelo de Gompertz (GMZ) existe também uma diferença significativa, determinada pelo teste de hipóteses onde a distribuição mais afastada da não diferença $\left(\mu_{\text {máx }}(\right.$ controle $)-\mu_{\text {máx }}($ tratamento $\left.)=0\right)$ entre os parâmetros da amostra controle $\mathrm{e}$ tratamento são consideradas diferentes, Figura 2. Observa-se ainda na Figura 2, que a distribuição das diferenças obtidas para as amostras tratadas com extrato supercrítico foram superiores aquelas tratadas com óleo essencial hidrodestilado, em comparação à amostra controle, isso se deve a diferença na composição química dos extratos $\mathrm{HD}$ e SC, indicando que o extrato supercrítico retardou mais o crescimento da BAC.

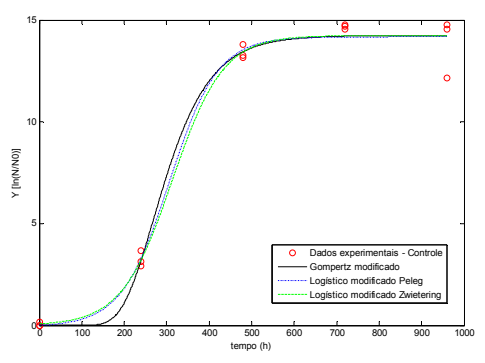

Controle

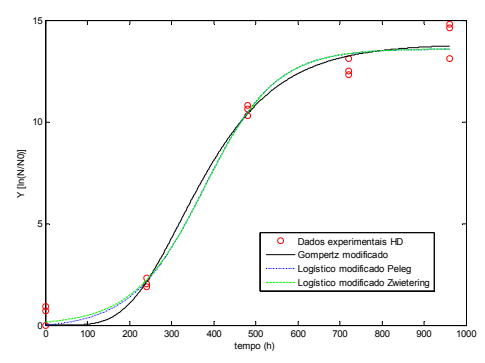

HD

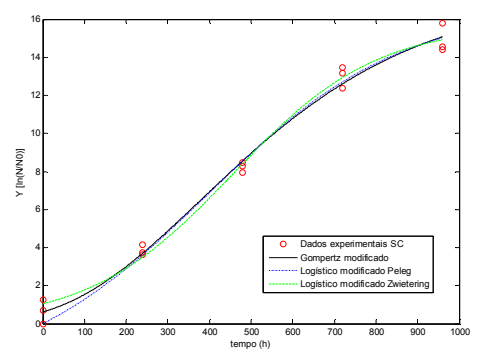

$\mathrm{SC}$

Figura 1 - Gráficos de comparação entre os ajustes pelos modelos GMZ, LMZ e LMCP aos dados das amostras controle, tratadas com óleo essencial hidrodestilado (HD) e tratadas com extrato supercrítico (SC), armazenados a $7^{\circ} \mathrm{C}$.

As mesmas análises foram realizadas para os outros modelos evidenciando uma mesma tendência nas comparações entre os tratamentos, reafirmando a importância da análise e sua validade na comparação. A estimativa da vida de prateleira do produto foi determinada no critério de $7 \log _{10}$ CFU. $g^{-1}$ de BAC, após esse critério foi considerado impróprio o consumo ou expirado o prazo de validade do mesmo. Para as amostras controle esse critério foi atingido aproximadamente no $18^{\circ}$ dia $(432 \mathrm{~h})$, e para as amostras tratadas com HD e SC, $28^{\circ}(672 \mathrm{~h})$ e $31^{\circ}(744 \mathrm{~h})$ dia de 
armazenagem.
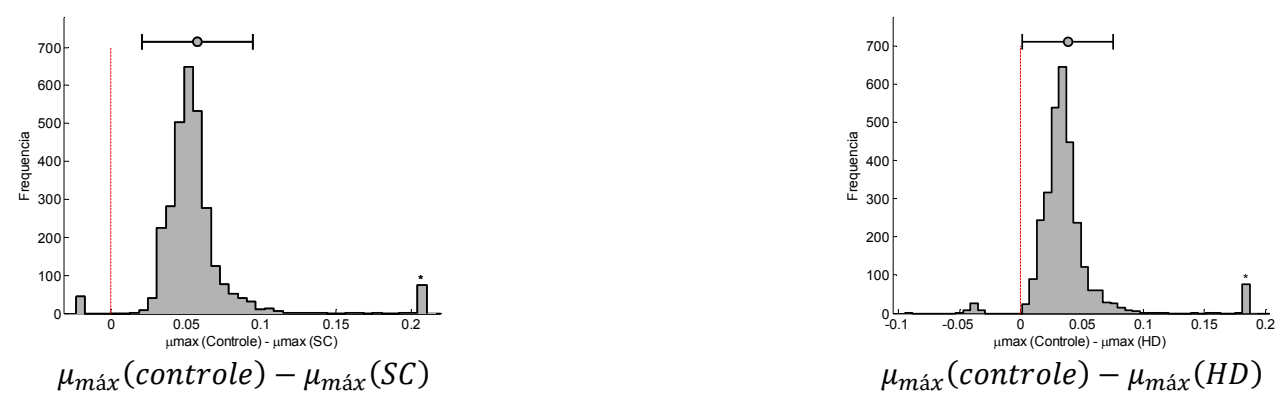

Figura 2 - Distribuição amostral do parâmetro $\mu_{\text {máx }}$ para as diferenças entre amostra controle e amostra tratada, obtido pelo modelo GMZ.

Pode-se ainda considerar o tempo da fase lag $\lambda$ ou $t_{c}$ outro parâmetro importante na manutenção da vida de prateleira. Porém para os tratamentos Controle, HD e SC a fase lag foi sendo reduzida, respectivamente, sendo a menor fase lag encontrada para o tratamento SC, aproximadamente $66 \%$ inferior à fase lag do Controle e HD. Porém para o modelo LMCP a fase lag aumento para os tratamentos Controle, HD e SC respectivamente, isso se deve a característica empírica dos parâmetros do modelo, sendo os valores encontrados pela regressão aqueles que minimizaram o erro em relação aos pontos experimentais. Dessa forma, pode-se supor a hipótese de não robustez do modelo (LMCP) em relação ao crescimento de bactérias láticas na presença de inibidores de crescimento, nesse caso os extratos de alecrim. Essa é uma avaliação importante, pois mesmo com o um bom ajuste, o mesmo não é adequado para a previsão de BAC na presença desse tipo de inibidor, ou seja os parâmetros encontrados não são comparáveis com os outros modelos, em termos físicos e biológicos.

Tabela 1 - Parâmetros dos modelos ajustados aos dados experimentais das amostras (armazenadas a $7{ }^{\circ} \mathrm{C}$ ) controle $(\mathrm{CON})$, tratadas com extrato hidrodestilado (HD) e com extrato supercítico (SC) de alecrim: os parâmetros, $\widehat{\boldsymbol{\theta}}_{b}^{*}$ foram estimados com as pseudo-amostras.

\begin{tabular}{|c|c|c|c|c|c|c|c|c|}
\hline & & \multicolumn{7}{|c|}{ Parâmetros Bootstrap $\hat{\theta}_{b}^{*}$} \\
\hline & & $A(\ln (N / N 0))$ & IC $(95 \%)$ & $\mu_{\text {máx }}\left(h^{-1}\right)$ & IC (95\%) & $\lambda(\boldsymbol{h})$ & IC (95\%) & $R^{2}$ \\
\hline \multirow{3}{*}{ CON } & GMZ & $14,053 \pm 1,734$ & {$[13,519 ; 14,645]$} & $0,079 \pm 0,037$ & {$[0,050 ; 0,226]$} & $195,594 \pm 15,510$ & {$[172,196 ; 226,180]$} & 0,989 \\
\hline & LMZ & $\begin{array}{c}14,258 \pm 0,254 \\
\boldsymbol{A}\end{array}$ & {$[13,647 ; 14,618]$} & $\begin{array}{c}0,065 \pm 0,027 \\
\boldsymbol{k}\end{array}$ & {$[0,047 ; 0,096]$} & $\begin{array}{c}194,655 \pm 12,668 \\
\boldsymbol{t}_{\boldsymbol{c}}\end{array}$ & {$[173,469 ; 227,491]$} & 0,989 \\
\hline & LMCP & $14,351 \pm 0,28$ & {$[13,685 ; 14,761]$} & $0,019 \pm 0,011$ & {$[0,013 ; 0,030]$} & $310,083 \pm 14,168$ & {$[277,471 ; 336,705]$} & 0,989 \\
\hline \multirow{3}{*}{ HD } & GMZ & $13,954 \pm 0,309$ & {$[13,367 ; 14,601]$} & $0,040 \pm 0,004$ & {$[0,034 ; 0,047]$} & $189,207 \pm 14,355$ & {$[161,045 ; 215,097]$} & 0,988 \\
\hline & LMZ & $\begin{array}{c}13,666 \pm 0,284 \\
\boldsymbol{A} \\
\end{array}$ & {$[13,117 ; 14,240]$} & $\begin{array}{c}0,040 \pm 0,003 \\
\boldsymbol{k} \\
\end{array}$ & {$[0,034 ; 0,047]$} & $\begin{array}{c}206,127 \pm 18,586 \\
\boldsymbol{t}_{\boldsymbol{c}} \\
\end{array}$ & {$[168,417 ; 239,673]$} & 0,988 \\
\hline & LMCP & $13,898 \pm 0,330$ & {$[13,272 ; 14,572]$} & $0,012 \pm 0,001$ & {$[0,009 ; 0,014]$} & $373,935 \pm 12,081$ & {$[350,128 ; 397,499]$} & 0,987 \\
\hline \multirow{3}{*}{ SC } & GMZ & $17,705 \pm 0,875$ & {$[16,227 ; 19,644]$} & $0,021 \pm 0,001$ & {$[0,019 ; 0,023]$} & $66,219 \pm 19,201$ & {$[26,473 ; 102,387]$} & 0,991 \\
\hline & LMZ & $\begin{array}{c}15,711 \pm 0,459 \\
\boldsymbol{A}\end{array}$ & {$[14,868 ; 16,655]$} & $\begin{array}{c}0,023 \pm 0,001 \\
\boldsymbol{k} \\
\end{array}$ & {$[0,021 ; 0,026]$} & $\begin{array}{c}113,879 \pm 19,720 \\
\boldsymbol{t}_{\boldsymbol{c}} \\
\end{array}$ & {$[74,475 ; 151,496]$} & 0,991 \\
\hline & LMCP & $19,375 \pm 0,871$ & {$[17,152 ; 20,000]$} & $0,005 \pm 0,000$ & {$[0,004 ; 0,006]$} & $391,096 \pm 23,245$ & {$[344,695 ; 433,946]$} & 0,989 \\
\hline
\end{tabular}

\section{CONCLUSÃO}

Pode-se concluir que a modelagem matemática do crescimento microbiano é uma ferramenta importante na compreensão do comportamento de algumas bactérias frente às condições ambientais para seu crescimento. Os tratamentos utilizados no PCF, ou modificações das condições ambientais 
para o crescimento das BAC resultaram em uma alteração nas suas taxas de crescimento e tempo de adaptação para sua reprodução. Conclui-se também que a técnica de bootstraping é uma ferramenta capaz de indicar o comportamento amostral dos parâmetros do modelo, a fim de auxiliar na análise paramétrica e estimação do seu intervalo de confiança. Observou-se através dessas análises que o PCF tratado com extrato SC apresentou uma menor taxa de crescimento da BAC segundo os modelos avaliados. Os três modelos se ajustaram bem aos dados experimentais, sendo válida a utilização de diferentes modelos para a melhor interpretação biológica do processo, onde se pôde verificar que o modelo LMCP não apresentou parâmetros compatível com esse sistema.

\section{REFERÊNCIAS}

CORRADINI, M. G.; PELEG, M. Estimating non-isothermal bacterial growth in foods from isothermal experimental data. Journal of applied microbiology, v. 99, n. 1, p. 187-200, jan. 2005.

DOWNES, F. P.; ITO, K.; ASSOCIATION, A. P. H. Compendium of Methods for the Microbiological Examination of Foods. [s.1.] American Public Health Association, 2001.

GENENA, A. K. et al. Rosemary (Rosmarinus officinalis): a study of the composition, antioxidant and antimicrobial activities of extracts obtained with supercritical carbon dioxide. Ciência e Tecnologia de Alimentos, v. 28, p. 463-469, 2008.

JAYASENA, D. D.; JO, C. Essential oils as potential antimicrobial agents in meat and meat products: A review. Trends in Food Science \& Technology, v. 34, n. 2, p. 96-108, dez. 2013.

LONGHI, D. A. et al. Assessing the prediction ability of different mathematical models for the growth of Lactobacillus plantarum under non-isothermal conditions. Journal of theoretical biology, v. 335, p. 88-96, 21 out. 2013.

SLONGO, A. P. et al. Modeling the growth of lactic acid bacteria in sliced ham processed by high hydrostatic pressure. LWT - Food Science and Technology, v. 42, n. 1, p. 303-306, jan. 2009.

THAI, H.-T. et al. A comparison of bootstrap approaches for estimating uncertainty of parameters in linear mixed-effects models. Pharmaceutical statistics, v. 12, n. 3, p. 129-40, 2013.

VERCAMMEN, A. et al. Shelf-life extension of cooked ham model product by high hydrostatic pressure and natural preservatives. Innovative Food Science \& Emerging Technologies, v. 12, n. 4, p. 407-415, out. 2011.

WÜST ZIBETTI, A. et al. Solvent extraction and purification of rosmarinic acid from supercritical fluid extraction fractionation waste: Economic evaluation and scale-up. The Journal of Supercritical Fluids, v. 83, p. 133-145, nov. 2013.

ZIBETTI, A. W.; BOLZAN, A.; MARANGONI, A. Antimicrobial activity and composition of rosemary (Rosmarinus Officinalis) extracts obtained with supercritical carbon dioxide pure and using ethanol as co-solvent. (INPL, Ed.)9th ISASF. Anais...Arcachon, France: ISASF, 2009

ZWIETERING, M. H. et al. Modeling of the bacterial growth curve. Applied and Environmental Microbiology, v. 56, n. 6, p. 1875-1881, 1990. 